\title{
The dialogue continues
}

\author{
The nanotoxicology community has numerous ideas and initiatives for improving the quality of \\ published papers.
}

In our Editorial last September ${ }^{1}$, we began a discussion on improving the quality and relevance of nanotoxicology research papers. This was followed by an open consultation and discussion at the 6th International Conference on Nanotoxicology in Beijing 2 . In light of this, we received a variety of feedback. We would like to thank all of our readers who provided comments, and we are publishing a number of these in this issue $e^{3-8,10}$.

With the comments we have chosen to publish, we have tried to highlight the range of opinions in the nanotoxicology community and illustrate some of the initiatives that are being developed to tackle the issue. We hope that publishing these comments will help

stimulate dialogue between individuals, laboratories and journals. Furthermore, at the time we went to press, comments were still arriving at the journal and we may publish some of these in subsequent issues.

Most of the readers who wrote in applauded the idea of implementing minimum characterization requirements and guidelines for reporting nanotoxicology studies in Nature Nanotechnology. However, few felt that this alone was enough to bring scientific rigour to the field. For example, members of the European NanoSafety Cluster initiative pointed out that having a minimum set of requirements is no substitute for rigorous peer review ${ }^{4}$; referees should still be looking closely at the methodology used in the paper and should request different types of information according to the purpose of the study. Similarly, members of the Hazardous Substances Advisory Committee in the UK commented that implementing consistent standards "starts with problem formulation and hypothesis generation", and that although standards implemented for regulatory purposes should be prescriptive, standards for research should be hypothesis-driven ${ }^{5}$. Furthermore, Kai Savolainen and Harri Alenius of the Finnish Institute of Occupational Health felt that short technical guidance documents on the safety of nanomaterials and technologies should be extended to other stakeholders such as regulators, decision makers, members of the innovation community and industrial partners ${ }^{6}$. This is because they believe that the importance of nanosafety on the societal and policy agenda needs to be stepped up.

Elsewhere, Warren Chan of the University of Toronto emphasized the need for a universally accepted, descriptive nomenclature system for describing and comparing nanomaterials in a nonbiased fashion ${ }^{7}$. Chan also calls for the creation of a database repository in which researchers can organize, analyse and compare data. This very issue has been the focus of the Nanotechnology Working Group of the US National Institutes of Health National Cancer Informatics Program, as is explained in the Correspondence by Nathan Baker and colleagues ${ }^{8}$. In particular, the working group has developed 'ISA-TAB-Nano', which provides a format for representing and sharing information about nanomaterials ${ }^{9}$.

In some of the contributions, specific points were raised about characterization methods and the immediate needs of the field. These contributions had several repeating themes and the key points are worth summarizing here. Specifically, there is agreement that more than one method should be used to characterize any parameter to ensure that measurements are unbiased. Furthermore, to facilitate comparisons between studies, the use of reference materials should quickly become common practice. Moreover, the reporting of dose should take into account the dynamic changes that can occur when nanomaterials are delivered to a living system or environment. In more specific technical comments, it was noted that dynamic light scattering should not be used to measure polydisperse, aggregated and non-spherical samples. Likewise, high-vacuum electron microscopy and spectroscopy techniques are inappropriate for hydrated and organic-rich samples. These techniques are probably the best of those currently available, but it is clear that there is an urgent need to develop new methods to characterize nanomaterials in the biological matrix.

A point was also made about the role of Nature Nanotechnology in this endeavour. Kenneth Dawson of University College Dublin suggests that role should be to continue publishing and promoting new ideas and discoveries that advance our understanding of this relatively new field; the policing of research reporting standards would be best left to more specialized journals ${ }^{10}$.

At Nature Nanotechnology, we have started a preliminary internal consultation (that may extend to other Nature Publishing Group journals) to take a decision on whether we should indeed implement reporting guidelines for authors and reviewing guidelines for referees, and propose the best way to do so. If we decide to implement such guidelines for nanotoxicology papers, we will outline our proposal in due course. In the meantime, the dialogue will continue outside the pages of the journal. For example, the topic is likely to be discussed at the upcoming conference in Prague organized by the European Unionfunded infrastructure, QNano ${ }^{11}$.

\footnotetext{
References

1. Nature Nanotech. 7, 545 (2012).

2. http://english.nanoctr.cas.cn/nanotoxicology 2012

3. Tantra, R. \& Shard, A. Nature Nanotech. 8, 71 (2013)

4. Fadeel, B. \& Savolainen, K. Nature Nanotech. 8, 71 (2013).

5. Lead, J. \& Holgate, S. Nature Nanotech. 8, 72 (2013).

6. Savolainen, K. \& Alenius, H. Nature Nanotech. 8, 72 (2013).

7. Chan, W. C. W. Nature Nanotech. 8, 72-73 (2013).

8. Baker, N. A. et al. Nature Nanotech. 8, 73-74 (2013).

9. http://nanoinformatics.org/2012/webinar/isa-tab-nano

10. Dawson, K. Nature Nanotech. 8, 73 (2013).

11. http://www.qnano-ri.eu/conference/welcome.html
} 